\title{
The Association of Non-Cardiac ECMO With Influenza Incidence: A Time Series Analysis
}

\author{
Michael Insel, Bhupinder Natt, Jarrod Mosier, Joshua Malo, and Christian Bime
}

\begin{abstract}
BACKGROUND: The 2009 H1N1 influenza epidemic saw a rise in the use of extracorporeal membrane oxygenation (ECMO) as a supportive therapy for refractory ARDS. We sought to determine whether ECMO utilization follows a seasonal pattern that matches the influenza season, and whether it can further be explained by the incidence of each influenza subtype. METHODS: We performed a longitudinal analysis of non-cardiac and cardiac-associated ECMO cases from the National In-patient Sample from 2005 to 2014, using overdispersed Poisson regression to evaluate associations with influenza incidence categorized by influenza-like illness and total positive influenza tests divided by subtype from the Centers for Disease Control and Prevention. RESULTS: Non-cardiac ECMO use was positively associated with influenza-like illness incidence in the current month (incidence risk ratio [IRR] 1.11, $95 \%$ confidence interval $[\mathrm{CI}] 1.07-1.15, P<.001$ ) and with influenza-like illness in the previous month (IRR 1.09, 95\% CI 1.05-1.14, $P<.001$ ). The 2009 H1N1 subtype had the strongest association with non-cardiac ECMO (IRR 1.19, 95\% CI 1.09-1.31, $P<.001)$. Cardiac ECMO was also positively associated with the incidence of influenza-like illness (IRR 1.05, 95\% CI 1.01-1.09, $P=.02$ ). CONCLUSION: Non-cardiac and cardiac ECMO use in the United States were significantly associated with influenza incidence. The influenza A, H1N1 2009, subtype had the strongest association. Key words: acute respiratory distress syndrome; influenza virus; ECMO; epidemiology; H1N1 virus; adults. [Respir Care 2019;64(3):279-284. (C 2019 Daedalus Enterprises]
\end{abstract}

\section{Introduction}

Extracorporeal membrane oxygenation (ECMO) is increasingly being used in refractory ARDS to rest the lungs as they recover from injury. ${ }^{1-3}$ There was a significant increase in ECMO use for ARDS in the United States during the $2009 \mathrm{H} 1 \mathrm{~N} 1$ influenza epidemic. ${ }^{4,5}$ The development of ARDS following H1N1 infection af-

Drs Insel, Natt, Malo, and Bime are affiliated with the Division of Pulmonary, Allergy, Critical Care \& Sleep Medicine, University of Arizona Health Sciences, Tucson, Arizona. Dr Mosier is affiliated with the Department of Emergency Medicine, University of Arizona College of Medicine, Tucson, Arizona.

The authors have disclosed no conflicts of interest.

Correspondence: Michael Insel MD, Division of Pulmonary, Allergy, Critical Care \& Sleep Medicine, University of Arizona College of Medicine, PO Box 245040-A, 1501 N. Campbell Ave., Tucson, AZ 85724. E-mail: minsel@deptofmed.arizona.edu.

DOI: $10.4187 /$ respcare.06145 fected young adults who were otherwise amenable to ECMO. 6,7 ECMO programs subsequently learned that they needed to be able to scale up quickly in anticipation of future outbreaks. ${ }^{8}$ Influenza has also been shown to increase the risk of myocardial infarction, ${ }^{9,10}$ which may further increase ECMO needs due to its downstream effects. Our ECMO program has since used the influenza season to anticipate our staffing and resource needs. It is unclear whether this is a useful strategy, or whether each influenza subtype portends unique risks for needing ECMO. We hypothesized that ECMO use for ARDS is associated with influenza incidence and that each influenza subtype has a unique association with ECMO use based on its potential in causing ARDS and myocardial infarction.

\section{Methods}

\section{Data Source}

Our analysis includes 3 datasets: The Agency for Healthcare Research and Quality Nationwide In-patient Sample 
(https://www.hcup-us.ahrq.gov/nisoverview.jsp, Accessed September 10, 2017), the Centers for Disease Control and Prevention (CDC) Out-patient Influenza-Like Illness Surveillance Network (ILINET), and the CDC Virologic Surveillance Program (https://www.cdc.gov/flu/weekly/index.htm, Accessed August 1, 2017).

The Nationwide In-patient Sample is part of the Healthcare Cost and Utilization Project (HCUP), Agency for Healthcare Research and Quality. The database represents a $20 \%$ stratified sample of all non-federal acute-care hospitalizations in the United States, representing 95\% of the total United States population. Hospitals submit discharge data voluntarily with 7 million hospital discharges included per year.

The ILINET database comprises weekly reported cases of influenza-like illness (ILI) by 2,900 out-patient health care providers in all 50 states. The CDC defines ILI as "fever and cough or sore throat in the absence of a known cause other than influenza" (https://www.cdc.gov/flu/ weekly/overview.htm\#Viral, Accessed July 17, 2017).

The CDC's influenza Virologic Surveillance Program includes 110 World Health Organization collaborating laboratories and 240 National Respiratory and Enteric Virus Surveillance System (WHO-NREVSS) laboratories across all 50 states. The laboratories report the total number of respiratory specimens tested, the percent positive, and the influenza subtype.

We identified hospitalizations in which ECMO was performed for adult patients, age $\geq 18 \mathrm{y}$, from 2005 to 2014. ECMO was identified by the International Classification of Diseases, 9th Revision procedure code 39.65. ECMO cases were divided into 2 categories-ie, non-cardiac ECMO and cardiac ECMO-to isolate ARDS-associated cases. The cardiac ECMO cases comprised all patients who underwent ECMO and carried a diagnosis of ventricular fibrillation or underwent ECMO and at least one of the following: valve surgery, coronary angiogram with or without stenting, coronary artery bypass graft, repair of a congenital heart defect, or heart transplant during the hospitalization. The non-cardiac ECMO cases compromised all other cases. The ECMO cases were obtained using each hospital's population weight within the HCUP sample.

The ILINET and WHO-NREVSS databases were obtained via the CDC's fluview web site (https://www. cdc.gov/flu/weekly/index.htm, Accessed August 15, 2017). The out-patient cases are reported weekly and comprise patients $25-64$ y old. The weekly data were averaged by the month so that they could be analyzed against the HCUP monthly ECMO data. Similarly, the WHO-NREVSS data were reported weekly and were analyzed as weekly averages per month.

\section{QUICK LOOK}

\section{Current knowledge}

Influenza incidence is strongly associated with ARDS and myocardial infarction. ECMO is increasingly utilized in severe ARDS to rest the lungs and in cardiogenic shock to unload the heart. The influenza H1N1 2009 epidemic saw a dramatic increase in ECMO utilization.

\section{What this paper contributes to our knowledge}

ECMO utilization is strongly associated with influenza incidence and specifically the incidence of the H1N1 2009 subtype. Influenza incidence and H1N1 incidence in the current month can be used to predict non-cardiac and cardiac ECMO needs in the next month.

\section{Primary Outcome}

The total number of non-cardiac ECMO and cardiac ECMO cases performed each month were our primary dependent variables. We performed 4 analyses for both outcome variables. First we looked at the association of ILI with noncardiac ECMO and cardiac ECMO in the current month. We then assessed the association of non-cardiac ECMO and cardiac ECMO with the ILI incidence in the previous month. We followed this analysis by associating non-cardiac ECMO and cardiac ECMO with the ILI 2 months earlier. Finally we assessed the association of non-cardiac ECMO and cardiac ECMO with each influenza subtype: AH1 pre-2009, AH1N1 2009, AH3, and B. The AH1N1 2009 subtype replaced the AH1N1 subtype after 2008. To account for this change, we included a dummy variable for each case after 2008.

\section{Statistical Analysis}

We performed a Poisson regression analysis with a scale parameter to accommodate extra-Poisson variation in the data. This overdispersion is modeled by a scale parameter estimated from model deviance (ie, the Pearson chi-square statistic divided by the residual degrees of freedom). ECMO utilization increased significantly from 2005 to 2014 . We adjusted for the increasing trend by including a double exponential smoothed linear trend variable using the tssmooth dexponential function in Stata (StataCorp, College Station, Texas). This procedure removes temporal trends using a geometrically weighted moving average filter. Month was included as a dummy variable to correct for persistent month effects across years. The results are presented as incidence rate ratios (IRRs) with 95\% confidence intervals (95\% CI), defined as the increase in ECMO incidence rate for every 1,000 more weekly influenza cases 
Table 1. Baseline Demographics

\begin{tabular}{|c|c|c|c|}
\hline & ECMO & Non-Cardiac ECMO & Cardiac ECMO \\
\hline Total & $17,951(-1329.49)$ & $8,146(-675.95)$ & $9,805(-717.54)$ \\
\hline \multicolumn{4}{|l|}{ Years } \\
\hline 2005 & $451(-172.4)$ & $267(-124.89)$ & $184(-59.34)$ \\
\hline 2006 & $363(-91.57)$ & $175(-60.87)$ & $188(-42.8)$ \\
\hline 2007 & $319(-119.41)$ & $110(-42.77)$ & $209(-83.42)$ \\
\hline 2008 & $909(-373.03)$ & $420(-161.57)$ & $489(-216.75)$ \\
\hline 2009 & $1,415(-465.22)$ & $576(-186.15)$ & $840(-293.19)$ \\
\hline 2010 & $1,410(-376.52)$ & $595(-179.36)$ & $816(-206.89)$ \\
\hline 2011 & $1,944(-570.7)$ & $999(-313.94)$ & $945(-274.45)$ \\
\hline 2012 & $2,725(-331.53)$ & $1,155(-160.3)$ & $1,570(-204.93)$ \\
\hline 2013 & $3,665(-446.07)$ & $1,640(-235.77)$ & $2,025(-242.79)$ \\
\hline 2014 & $4,750(-513.27)$ & $2,210(-289.36)$ & $2,540(-263.03)$ \\
\hline Mean age, $y$ & $51.4(-0.33)$ & $45.9(-0.48)$ & $55.9(-0.37)$ \\
\hline Female & $38 \%(-0.01)$ & $43 \%(-0.01)$ & $34 \%(-0.01)$ \\
\hline \multicolumn{4}{|l|}{ Race } \\
\hline White & $58 \%(-0.02)$ & $57 \%(-0.02)$ & $59 \%(-0.02)$ \\
\hline African-American & $12 \%(-0.01)$ & $14 \%(-0.01)$ & $11 \%(-0.01)$ \\
\hline Hispanic & $7 \%(-0.01)$ & $7 \%(-0.01)$ & $7 \%(-0.01)$ \\
\hline Asian & $2 \%$ (NA) & $1 \%$ (NA) & $2 \%$ (NA) \\
\hline Native American & $1 \%(\mathrm{NA})$ & $1 \%(\mathrm{NA})$ & $1 \%(\mathrm{NA})$ \\
\hline Not listed & $14 \%(-0.02)$ & $14 \%(-0.02)$ & $14 \%(-0.03)$ \\
\hline Other & $6 \%(-0.01)$ & $6 \%(-0.01)$ & $6 \%(-0.01)$ \\
\hline Length of stay, d & $23.79(-0.68)$ & $22.83(-1.01)$ & $24.96(-0.83)$ \\
\hline Mortality & $57 \%(-0.01)$ & $55 \%(-0.02)$ & $58 \%(-0.02)$ \\
\hline \multicolumn{4}{|c|}{$\begin{array}{l}\text { Values are presented as } n \text { (standard error) or \% (standard error). } \\
\text { ECMO = extracorporeal membrane oxygenation } \\
\text { NA }=\text { not applicable }\end{array}$} \\
\hline
\end{tabular}

per month, or 4,000 monthly cases. A $P$ value $<.05$ was considered statistically significant. ${ }^{11,12}$

\section{Results}

\section{Baseline}

There were an estimated 17,951 ECMO cases, standard error (SE) 1,329, from 2005 to 2014 . Among these, 8,146 cases (SE 676) were non-cardiac ECMO and 9,805 cases ( $\mathrm{SE}=$ 718) were cardiac ECMO cases. The non-cardiac ECMO cases increased from 267 in 2005 to 2,210 in 2014. Among the non-cardiac ECMO cohort, $43 \%$ were women, $57 \%$ white, $14 \%$ African-American, and 7\% Hispanic. The mean age was 46 y for non-cardiac ECMO and 56 y for cardiac ECMO; $55 \%$ of non-cardiac ECMO patients died compared to $58 \%$ of the cardiac ECMO patients (Table 1).

\section{Non-Cardiac ECMO and Influenza-Like Illnesses}

From January 2005 to December 2014, non-cardiac ECMO use per month was significantly associated with ILI (IRR 1.11, 95\% CI 1.07-1.15, $P<.001$ ) (Fig. 1).
Average weekly ILI cases during the previous month were also associated with the current month's ECMO use (IRR 1.09, 95\% CI 1.05-1.14, $P<.001$ ). The average weekly cases 2 months prior was not significantly associated with the current month's non-cardiac ECMO cases (IRR 1.05, 95\% CI 1.00-1.10) (Table 2).

\section{Non-Cardiac ECMO and Influenza Subtype}

The influenza A H1N1 2009 subtype was strongly associated with non-cardiac ECMO (IRR 1.19, 95\% CI 1.09$1.31, P<.001)$. Prior to 2009 , the AH1N1 subtype was negatively associated with non-cardiac ECMO (IRR 0.20, 95\% CI 0.06-0.67). Influenza B and AH3 had a positive association with non-cardiac ECMO but were not statistically significant (influenza B: IRR 1.15, 95\% CI $0.84-$ 1.57; AH3: IRR 1.07, 95\% CI 0.96-1.20) (Fig. 2).

\section{Non-Cardiac ECMO and Influenza Subtype in the Previous Month}

The average number of influenza A H1N1 2009 cases in the previous month was also associated with the cur- 


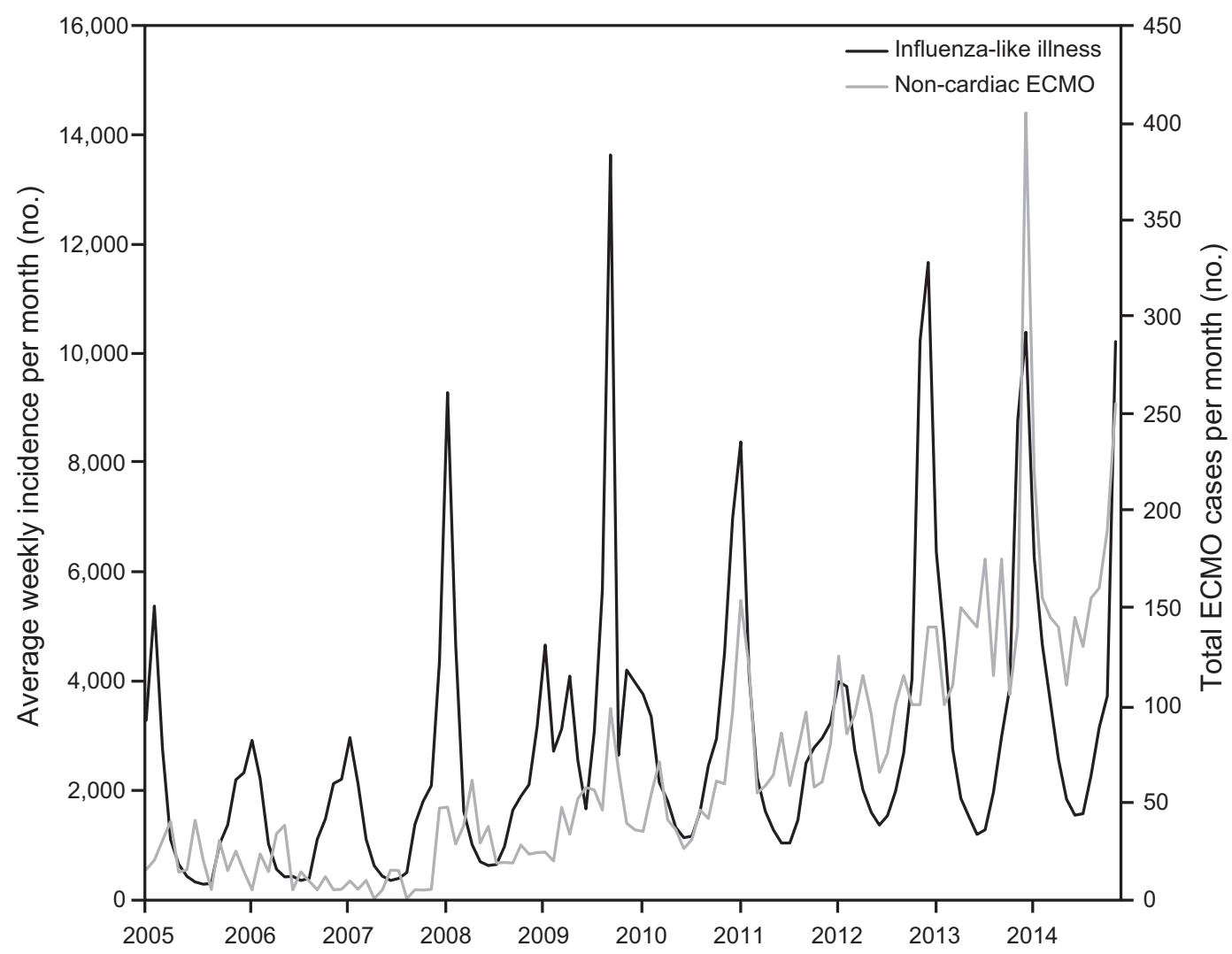

Fig. 1. Average weekly influenza-like illnesses per month and monthly non-cardiac extracorporeal membrane oxygenation (ECMO) cases.

Table 2. Association of CE and non-cardiac ECMO with ILI

\begin{tabular}{lcccc}
\hline \hline & $\begin{array}{c}\text { Incidence } \\
\text { Risk } \\
\text { Ratio }\end{array}$ & $\begin{array}{c}\text { Standard } \\
\text { Error }\end{array}$ & $P$ & $95 \%$ CI \\
& & & & \\
\hline Non-cardiac ECMO & 1.11 & 0.02 & $<.001$ & $1.07-1.15$ \\
ILI in current month* & 1.09 & 0.02 & $<.001$ & $1.05-1.14$ \\
In previous month* & 1.05 & 0.02 & .06 & $1.00-1.10$ \\
2 months earlier & & & & \\
Cardiac ECMO & 1.05 & 0.02 & .02 & $1.01-1.09$ \\
ILI in current month* & 1.05 & 0.02 & .02 & $1.01-1.09$ \\
In previous month* & 1.03 & 0.02 & .20 & $0.99-1.07$ \\
2 months earlier & & &
\end{tabular}

Each row represents a separate Poisson analysis with the corresponding controls and variables: the monthly dummy variable and trend variable.

* Statistically significant result.

$\mathrm{CE}=$ cardiac ECMO

$\mathrm{ECMO}=$ extracorporeal membrane oxygenation

ILI = influenza-like illness

rent month's non-cardiac ECMO cases (IRR 1.19, 95\% CI 1.07-1.32, $P<.01)$, indicating H1N1 incidence in the current month can help predict the non-cardiac ECMO use in the next month. The previous month's incidence of the other subtypes were not significantly correlated with non-cardiac ECMO (Table 3).

\section{Cardiac ECMO and Influenza Incidence}

Previous studies have demonstrated that influenza may trigger cardiac events. 9,10 Cardiac ECMO was significantly associated with ILI (IRR 1.05, 95\% CI 1.01-1.09, $P=.02$ ). Cardiac ECMO was also associated with the ILI in the previous month (IRR $1.05,95 \%$ CI 1.01-1.09, $P=.02$ ). The influenza subtypes in the current and previous month were not associated with cardiac ECMO (Table 3).

\section{Discussion}

Our results confirm that non-cardiac ECMO and cardiac ECMO use are independently associated with influenza incidence. The H1N1 2009 subtype had the strongest association with non-cardiac ECMO use. We were further able to show that influenza incidence in the previous month, specifically influenza H1N1 2009 subtype incidence, was associated with increased use of ECMO in the current month. ECMO programs can use current influenza events, specifically H1N1 events, to help predict ECMO use in the following month. Both influenza $\mathrm{B}$ and $\mathrm{AH} 3$ were not significantly associated with ECMO use. These results either reflect the coincidence of influenza $\mathrm{B}$ and $\mathrm{AH} 3$ diluting their relative effect or the lower virulence of each 


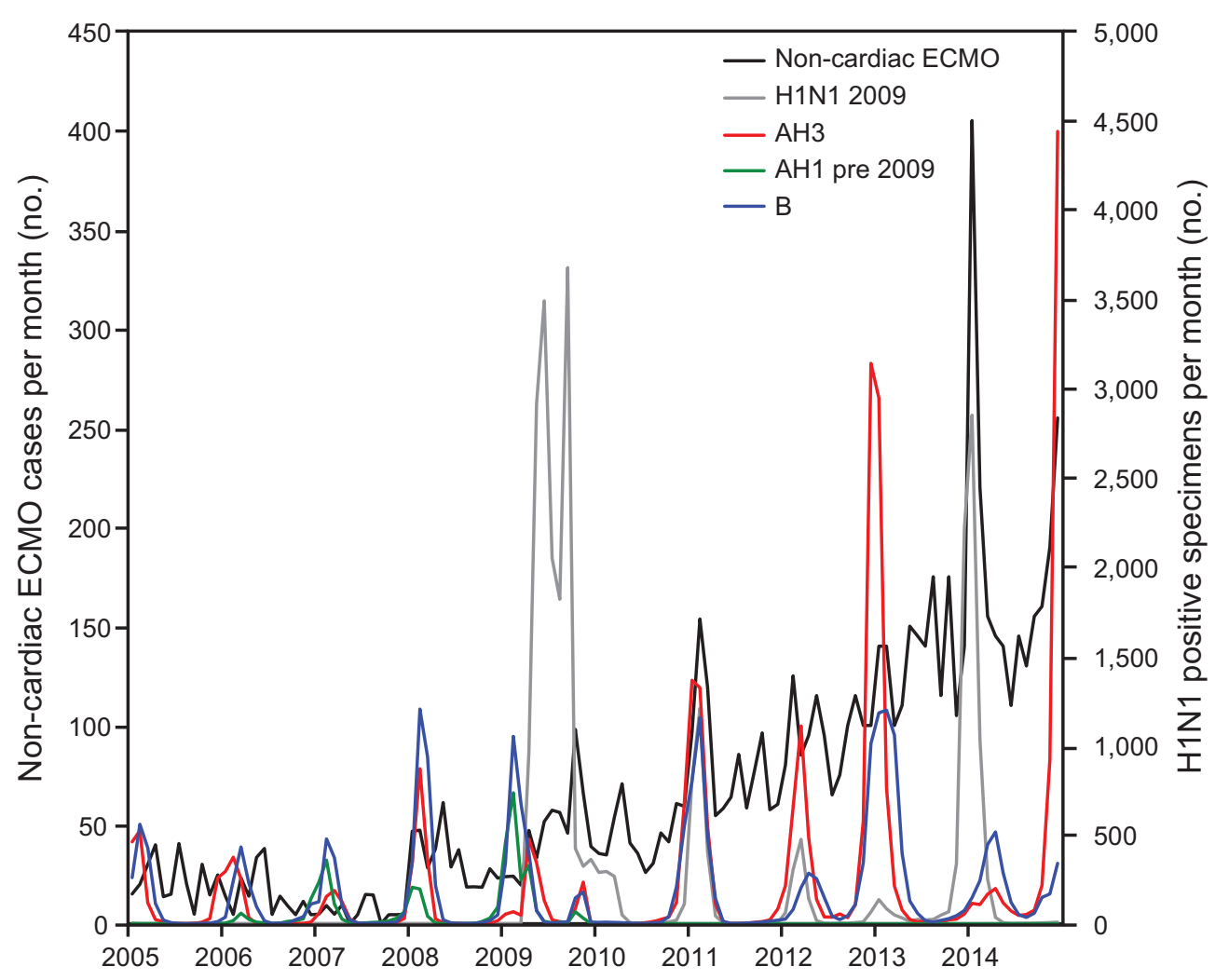

Fig. 2. Monthly trend in non-cardiac extracorporeal membrane oxygenation (ECMO) and weekly average influenza cases separated by subtype. AH1 represents the influenza A H1N1 subtype. After 2009, the Centers for Disease Control and Prevention replaced the generic subtype with H1N1 2009.

subtype compared to the H1N1 2009 subtype. The results provide further evidence that the H1N1 2009 subtype portends a greater risk for ARDS among patients who are amenable ECMO candidates as compared to the other influenza subtypes.

The primary strength of our study is that its sample reflects what has happened nationally by using the Nationwide In-patient Sample and CDC databases. In addition, by including a trend variable we were able to minimize the effect that greater provider familiarity with ECMO and increased ECMO popularity over the 10-y period had on ECMO use. By including a month dummy variable, we reduced the effect that seasonal illnesses, which often occur in concordance with influenza, had on ECMO use.

Our analysis has many limitations. The use of multiple data sets creates some statistical challenges. Our analysis required 2 estimation steps, which can lead to standard errors that are greater than the presented standard errors. The HCUP dataset is a stratified sample of hospitalizations. We used this sample to obtain estimates for the total population and then analyzed these estimates with influenza incidence. Using the estimates, which have their own standard errors, makes the standard errors calculated in our regression analysis less than would be calculated if we used a single dataset.
Another limitation is that the HCUP data and CDC data are gathered by hospitals and provider self-reports. They may not actually reflect the true number of influenza or ECMO cases. Based on our previous publications, we believe that HCUP procedural codes are an accurate way to create a cohort. ${ }^{5}$

The ILI database requires out-patient providers to report to the CDC. The criteria for ILI may not be specific enough, and the demands for self-report may be too great to capture the true incidence of out-patient influenza. In addition, ILI may in fact be a surrogate for all viral respiratory illnesses, which could be a surrogate for ARDS risk and a general need for ECMO. Likewise, the virologic surveillance data requires a clinician to have enough pretest probability to send influenza specimens.

Another potential limitation is that we defined non-cardiac ECMO by excluding cardiac procedures rather than looking at the difference between venovenous and venoarterial ECMO. Venovenous ECMO may be a better way to identify non-cardiac ECMO. We chose not to divide these groups by cannulation type due to inadequate and incomplete reporting in the HCUP database.

Finally, because our study is a retrospective study using more than one data source, we are unable to provide a relative risk of influenza causing ARDS severe enough to 
Table 3. Association of CE and non-cardiac ECMO with Influenza Subtype

\begin{tabular}{|c|c|c|c|c|}
\hline & $\begin{array}{c}\text { Incidence } \\
\text { Risk } \\
\text { Ratio }\end{array}$ & $\begin{array}{l}\text { Standard } \\
\text { Error }\end{array}$ & $P$ & $95 \% \mathrm{CI}$ \\
\hline \multicolumn{5}{|c|}{ NCE and influenza subtype } \\
\hline H1N1 pre-2009 & 0.20 & 0.12 & .01 & $0.06-0.67$ \\
\hline H1N1 2009 & 1.19 & 0.06 & $<.001$ & $1.09-1.31$ \\
\hline $\mathrm{AH} 3$ & 1.07 & 0.61 & .23 & $0.96-1.19$ \\
\hline $\mathrm{B}$ & 1.14 & 0.18 & .38 & $0.84-1.57$ \\
\hline \multicolumn{5}{|c|}{$\begin{array}{l}\text { NCE and influenza in } \\
\text { previous month }\end{array}$} \\
\hline H1N1 pre-2009 & 0.21 & 0.15 & .03 & $0.05-0.84$ \\
\hline H1N1 2009 & 1.19 & 0.05 & $<.001$ & $1.09-1.30$ \\
\hline $\mathrm{AH} 3$ & 1.03 & 0.09 & .73 & $0.86-1.24$ \\
\hline B & 1.18 & 0.24 & .44 & $0.78-1.77$ \\
\hline \multicolumn{5}{|c|}{$\mathrm{CE}$ and influenza subtype } \\
\hline H1N1 pre-2009 & 1.10 & 0.43 & .80 & $0.51-2.36$ \\
\hline H1N1 2009 & 1.02 & 0.05 & .69 & $0.93-1.11$ \\
\hline $\mathrm{AH} 3$ & 0.98 & 0.05 & .64 & $0.88-1.08$ \\
\hline $\mathrm{B}$ & 1.13 & 0.17 & .39 & $0.85-1.51$ \\
\hline \multicolumn{5}{|l|}{$\begin{array}{l}\mathrm{CE} \text { and influenza in } \\
\text { previous month }\end{array}$} \\
\hline H1N1 pre-2009 & 1.00 & 0.43 & $>.99$ & $0.43-2.34$ \\
\hline H1N1 2009 & 1.01 & 0.06 & .75 & $0.93-1.11$ \\
\hline $\mathrm{AH} 3$ & 1.01 & 0.08 & .90 & $0.86-1.19$ \\
\hline B & 1.06 & 0.19 & .74 & $0.75-1.49$ \\
\hline $\begin{array}{l}\mathrm{CE}=\text { cardiac ECMO } \\
\mathrm{NCE}=\text { non-cardiac ECM } \\
\mathrm{ECMO}=\text { extracorporeal } \\
\mathrm{ILI}=\text { influenza-like illnes }\end{array}$ & xygenation & & & \\
\hline
\end{tabular}

need ECMO or demonstrate that ECMO provides a clinical benefit.

Future studies that look at patient-specific factors and ECMO utilization are needed to demonstrate how age, demographics, and other biologic factors drive the decision to initiate ECMO. Additional studies are needed to examine the clinical benefit of ECMO in the setting of influenza-associated ARDS.

\section{Conclusion}

Non-cardiac ECMO was independently associated with influenza incidence between 2005 and 2014. Influenza A
H1N1 2009 subtype had the strongest association. These results confirm that influenza incidence, and specifically H1N1 incidence, can be used to help ECMO programs anticipate their staffing and hospital resource needs.

\section{ACKNOWLEDGMENTS}

We thank Dr Dean Billheimer for support with statistical analysis.

\section{REFERENCES}

1. Peek GJ, Mugford M, Tiruvoipati R, Wilson A, Allen E, Thalanany $\mathrm{MM}$, et al. Efficacy and economic assessment of conventional ventilatory support versus extracorporeal membrane oxygenation for severe adult respiratory failure (CESAR): a multicentre randomised controlled trial. Lancet 2009;374(9698):1351-1363.

2. Lindskov C, Jensen RH, Sprogoe P, Klaaborg KE, Kirkegaard H, Severinsen IK, et al. Extracorporeal membrane oxygenation in adult patients with severe acute respiratory failure. Acta Anaesthesiol Scand 2013;57(3):303-311.

3. Combes A, Bacchetta M, Brodie D, Müller T, Pellegrino V. Extracorporeal membrane oxygenation for respiratory failure in adults. Curr Opin Crit Care 2012;18(1):99-104.

4. Davies A, Jones D, Bailey M, Beca J, Bellomo R, Blackwell N, et al. Extracorporeal membrane oxygenation for 2009 influenza A(H1N1) acute respiratory distress syndrome. JAMA 2009;302(17):1888-1889.

5. Natt BS, Desai H, Singh N, Poongkunran C, Parthasarathy S, Bime C. Extracorporeal membrane oxygenation for ARDS: national trends in the United States 2008-2012. Respir Care 2016;61(10):1293-1298.

6. Lagacé-Wiens PR, Rubinstein E, Gumel A. Influenza epidemiology: past, present, and future. Crit Care Med 2010;38:e1-e9.

7. Kumar A, Zarychanski R, Pinto R, Cook DJ, Marshall J, Lacroix J, et al.; Canadian Critical Care Trials Group H1N1 Collaborative. Critically ill patients with 2009 influenza A(H1N1) infection in Canada. JAMA 2009;302(17):1872-1879.

8. Michaels AJ, Hill JG, Long WB, Young BP, Sperley BP, Shanks TR, Morgan LJ. Adult refractory hypoxemic acute respiratory distress syndrome treated with extracorporeal membrane oxygenation: the role of a regional referral center. Am J Surg 2013;205(5):492-498.

9. Warren-Gash C, Bhaskaran K, Hayward A, Leung GM, Lo SV, Wong CM, et al. Circulating influenza virus, climatic factors, and acute myocardial infarction: a time series study in England and Wales and Hong Kong. J Infect Dis 2011;203(12):1710-1708.

10. Kwong J, Schwartz K, Campitelli M, Chung H, Crowcroft N, Karnauchow $\mathrm{T}$, et al. Acute myocardial infarction after laboratory-confirmed influenza infection. N Engl J Med 2018;378(4):345-353.

11. Lachin J. Biostatistical methods: the assessment of relative risk, 2nd ed. Hoboken, NJ: Wiley; 2010, 382-427.

12. Frome EL, Checkoway H. Epidemiologic programs for computers and calculators: use of Poisson regression models in estimating incidence rate and ratios. Am J Epidemiology 1985;121(2):309-323. 